\title{
The discovery of high-amplitude, 10.9-minute oscillations in the cool magnetic Ap star HD 115226 ${ }^{\star}$
}

\author{
O. Kochukhov $^{1}$, T. Ryabchikova ${ }^{2,3}$, S. Bagnulo ${ }^{4}$, and G. Lo Curto ${ }^{5}$ \\ 1 Department of Astronomy and Space Physics, Uppsala University, 75120 Uppsala, Sweden \\ e-mail: oleg@astro.uu.se \\ 2 Institute of Astronomy, Russian Academy of Sciences, Pyatnitskaya 48, 109017 Moscow, Russia \\ 3 Department of Astronomy, University of Vienna, Türkenschanzstraße 17, 1180 Vienna, Austria \\ 4 Armagh Observatory, College Hill, Armagh BT61 9DG, Northern Ireland \\ 5 European Southern Observatory, Alonso de Cordova 3107, Vitacura, Santiago, Casilla 19001 Santiago 19, Chile
}

Received 3 December 2007/ Accepted 19 December 2007

\section{ABSTRACT}

\begin{abstract}
We present the discovery of pulsational variations in the cool magnetic Ap star HD 115226 - the first high-amplitude rapidly oscillating Ap (roAp) star discovered with time-series spectroscopy. Using high-resolution spectra obtained with the HARPS instrument at the European Southern Observatory 3.6-m telescope, we detect radial velocity variations with a period of $10.86 \mathrm{~min}$ in $\mathrm{Pr}$ III, Nd III, Dy III lines, and in the narrow cores of hydrogen lines. Pulsational amplitudes exceed $1 \mathrm{~km} \mathrm{~s}^{-1}$ in individual lines of Nd III. The presence of running waves in the stellar atmosphere is inferred from a phase shift between the radial velocity maxima of rare-earth and hydrogen lines. Our abundance analysis demonstrates that HD 115226 exhibits a typical roAp spectroscopic signature, notably ionization anomaly of $\mathrm{Pr}, \mathrm{Nd}$, and Dy. We discuss the discovery of pulsations in HD 115226 in the context of recent spectroscopic studies of roAp stars and point to the existence of a correlation between spectroscopic pulsational amplitude and the stellar rotation rate.
\end{abstract}

Key words. stars: atmospheres - stars: chemically peculiar - stars: oscillations - stars: individual: HD 115226

\section{Introduction}

Rapidly oscillating Ap (roAp) stars are cool, magnetic chemically-peculiar stars pulsating in high-overtone non-radial $p$-modes with periods around 10 min. The excitation of oscillations in these stars is closely related to the presence of strong global magnetic fields, as the pulsational axis is seen to be aligned with the axis of oblique magnetic fields and pulsations are enhanced at the magnetic poles (Kochukhov 2004). In addition to remarkable pulsation behaviour, which opens interesting prospects for asteroseismology (Vauclair \& Théado 2004), roAp stars are characterized by extremely anomalous surface chemical composition (Ryabchikova et al. 2004) as well as strongly inhomogeneous horizontal and vertical distribution of chemical elements (Kochukhov et al. 2004; Ryabchikova et al. 2002).

Most of the previous work on identification and frequency analysis of the three dozen currently known roAp stars was performed using time-series photometry at small telescopes (Kurtz $\&$ Martinez 2000). However, inspired by the discovery of outstanding pulsational variations in the lines of rare-earth elements (REEs) (Savanov et al. 1999; Kochukhov \& Ryabchikova 2001), the focus of observational studies of roAp stars is shifting toward time-resolved spectroscopy. A number of recent studies (e.g., Mkrtichian et al. 2003; Ryabchikova et al. 2007b) have demonstrated the unique diagnostic potential of the precise radial velocity and line profile measurements in individual lines.

\footnotetext{
* Based on observations collected at the European Southern Observatory, La Silla, Chile (ESO program 079.D-0118).
}

This type of observational material allows one to trace propagation of pulsation waves through the chemically-stratified stellar atmosphere, eventually obtaining a three-dimensional picture of pulsations and chemical inhomogeneities (Kochukhov 2007a).

Despite these noteworthy achievements of time-resolved spectroscopic studies, relatively little has been done in the direction of expanding the list of known roAp stars and understanding the coexistence of pulsating and apparently constant Ap stars in the same region of the H-R diagram. With the aim to obtain an improved picture of the incidence of pulsations in Ap stars, we have initiated a survey of cool peculiar magnetic stars with the ultra-stable High Accuracy Radial velocity Planet Searcher (HARPS) spectrograph at the European Southern Observatory (ESO). Here we report the first result of our observations - the discovery of high-amplitude oscillations in the magnetic Ap star HD 115226.

\section{Observations and data reduction}

We observed HD 115226 on the night of April 15, 2007, with the HARPS spectrograph (Mayor et al. 2003) at the ESO 3.6-m telescope at La Silla. The observations have started at the barycentric JD 2454205.63472 and continued for $4.3 \mathrm{~h}$. In total, we collected 102 consecutive $120 \mathrm{~s}$ exposures. The dead time between stellar observations was $31 \mathrm{~s}$.

We carried out the extraction of one-dimensional spectra and barycentric velocity correction of the wavelength scale with the HARPS pipeline available at the telescope. We achieved the peak signal-to-noise ratio of 40-45 in the individual exposures of 
HD 115226. The spectra have resolving power $\lambda / \Delta \lambda=115000$, and cover a wavelength range from 3780 to $6910 \AA$.

The dominant source of radial velocity noise in our data is due to the photon noise rather than to the instrumental precision. Therefore, we did not use the simultaneous ThAr method, avoiding in this way any contamination of the stellar signal. At the beginning and at the end of HD 115226 observations we took a ThAr reference spectrum, and we estimate that the instrumental drift within the time series was at most $0.36 \mathrm{~m} \mathrm{~s}^{-1}$. Following the procedure outlined in Kochukhov et al. (2007b), we postprocessed all extracted spectra of HD 115226 with the aim to achieve an accurate and consistent continuum normalization.

\section{Basic properties of HD 115226}

Little is known about HD 115226 (HIP 64883, CP-72 1373). This southern $V=8.51$ chemically-peculiar star is classified as A3p Sr in the General Catalogue of Ap and Am Stars (Renson et al. 1991). Gomez et al. (1998) and Kochukhov \& Bagnulo (2006) included this object in their studies of the evolutionary state of magnetic Ap stars. Using Hipparcos parallax and $T_{\text {eff }}=7640 \mathrm{~K}$ estimated from the Geneva photometric colors, the authors of the latter paper determined $L=7.2 \pm 1.9 L_{\odot}$. From the comparison with standard stellar evolutionary models (Schaller et al. 1992) one can infer that these parameters correspond to a $M=1.60 \pm 0.05 M_{\odot}$ star with a fractional age between 0.0 and 0.4 of the main sequence lifetime.

The Strömgren photometry of HD 115226 (Martinez 1993) indicates $T_{\text {eff }}=8000-8200 \mathrm{~K}$ if normal-star calibrations of Moon \& Dworetsky (1985) and Napiwotzki et al. (1993) are used. However, comparison of the theoretical calculations with the observed profiles of $\mathrm{H} \alpha$ and $\mathrm{H} \beta$ favours lower $T_{\text {eff }}$ suggested by the Geneva photometric parameters.

Kochukhov \& Bagnulo (2006) determined longitudinal field $\left\langle B_{z}\right\rangle=752 \pm 48 \mathrm{G}$ from a single circular polarization measurement of HD 115226 with the FOcal Reducer and Spectrograph (FORS1) at the ESO Very Large Telescope. This robust magnetic field detection, along with the typical peculiar-star appearance of the spectrum of HD 115226 (Fig. 1), leaves no doubt that this star belongs to the group of cool magnetic Ap stars. The value of $T_{\text {eff }}$ inferred for HD 115226 is also typical of the known roAp stars.

To perform a preliminary analysis of the atmospheric chemistry of HD 115226 and to compare it with the chemical abundances of well-studied roAp stars, we adopted a model atmosphere of the roAp star 10 Aql (HD 176232) with the parameters $T_{\text {eff }}=7650 \mathrm{~K}, \log g=4.0$, determined in our study of Ca stratification in 10 Aql (Ryabchikova et al. 2007a). A rather rapid rotation of HD 115226 does not allow us to study individual lines for most elements, therefore we performed magnetic spectral synthesis with the help of SYNTHMAG code (Kochukhov 2007b). First of all, we tried to estimate $v_{\mathrm{e}} \sin i$ and the strength of surface magnetic field using magnetic intensification. Line profiles of almost all elements in the spectrum of HD 115226 have complex structures, indicating a high degree of horizontal abundance inhomogeneities. Best-fit projected rotational velocities vary from $18 \mathrm{~km} \mathrm{~s}^{-1}$ for Co I and Y II to $23-25 \mathrm{~km} \mathrm{~s}^{-1}$ for REEs and Si II lines, and up to $28-30 \mathrm{~km} \mathrm{~s}^{-1}$ for $\mathrm{Mg}, \mathrm{Ca}, \mathrm{Cr}$, and $\mathrm{Fe}$ lines. This dispersion in broadening is accompanied by different shifts of the line centroids: $-10 \mathrm{~km} \mathrm{~s}^{-1}$ for Co I, from -5 to $-8 \mathrm{~km} \mathrm{~s}^{-1}$ in REEs, from -2 to $-3 \mathrm{~km} \mathrm{~s}^{-1}$ in $\mathrm{Mg}, \mathrm{Ca}, \mathrm{Cr}, \mathrm{Fe}$, and $+7 \mathrm{~km} \mathrm{~s}^{-1}$ for $\mathrm{Si}$. These discrepancies point to a spotted distribution of chemical elements at the stellar surface.

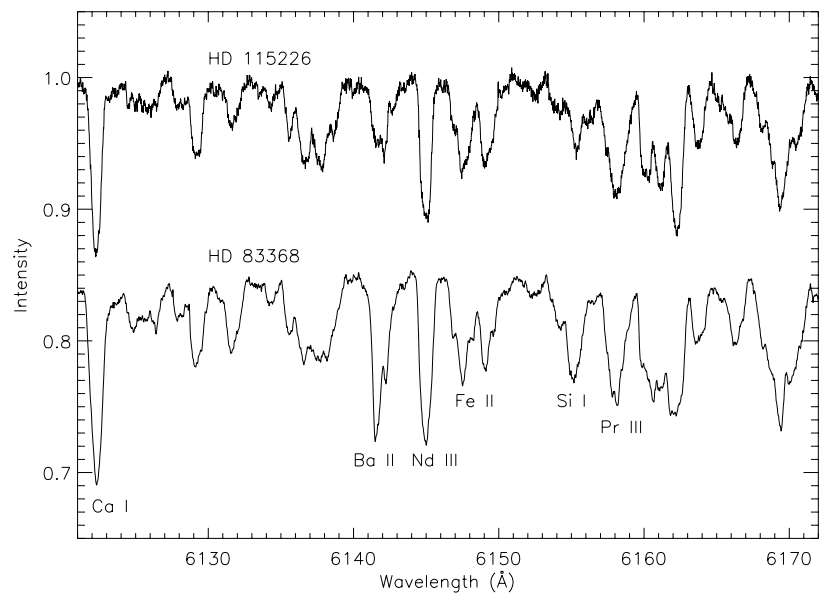

Fig. 1. Comparison of the 6120-6170 $\AA$ region in the average spectrum of HD 115226 with the known rapidly rotating roAp star HD 83368 (Kochukhov et al. 2004). The spectrum of HD 83368 is shifted down for display purposes.

Table 1. Chemical abundances in the atmosphere of HD 115226. Error estimates are given for species with sufficient number of measured lines. In other cases, we provide a range of abundance that fits individual spectral features.

\begin{tabular}{ll|ll}
\hline \hline Ion & $\log \left(N_{\text {el }} / N_{\text {tot }}\right)$ & Ion & $\log \left(N_{\text {el }} / N_{\text {tot }}\right)$ \\
\hline $\mathrm{Mg}$ I/II & -4.5 & Ce II & -9.5 \\
Si I/II & -4.6 & Pr II & -9.6 \\
Ca I/II & $-5.4 \pm 0.2$ & Pr III & $-8.1 \pm 0.2$ \\
Ti II & -7.0 & Nd II & -8.8 to -9.0 \\
Cr I/II & -5.0 & Nd III & $-7.2 \pm 0.3$ \\
Fe I/II & -4.5 to -4.6 & Sm II & -9.2 to -9.5 \\
Ni I & -6.3 & Eu II & -10.2 \\
Co I & -6.0 & Gd II & -9.3 \\
Y II & -8.5 & Tb III & -8.2 \\
Ba II & -10.5 & Dy II & -9.0 \\
La II & -9.50 & Dy III & $-7.3 \pm 0.2$ \\
\hline
\end{tabular}

We performed spectrum synthesis for the magnetic field strength in the range from 1 to $3 \mathrm{kG}$. We obtained the lowest dispersion in the abundances inferred from spectral lines with different Landé factors (0-2.5 for Fe lines and 0.6-1.6 for $\mathrm{Nd}$ III lines) and different Zeeman structure for the magnetic field strength of $1 \mathrm{kG}$.

The result of abundance analysis of HD 115226 is summarized in Table 1. The inferred abundances are typical of roAp stars in the 7500-8000 K effective temperature range. The star has nearly solar concentrations of $\mathrm{Mg}, \mathrm{Si}, \mathrm{Ti}$, and $\mathrm{Fe}$, an overabundance of $\mathrm{Cr}$ and $\mathrm{Co}$, and an underabundance of Ba. The well-known REE anomaly, where abundance derived from the lines of second ions (Pr III, Nd III, Dy III) exceeds by 1.5-2 dex those derived from the lines of singly-ionized species (Ryabchikova et al. 2001, 2004), is also present. Interestingly, the vertical $\mathrm{Ca}$ abundance distribution determined for the atmosphere of $10 \mathrm{Aql}$ fits well the Ca line profiles in the spectrum of HD 115226. This suggests close similarity of the Ca stratifications in the atmospheres of these two stars.

Significant Doppler broadening of the HD 115226 spectrum indicates that the stellar rotational period is short. Combining $v_{\mathrm{e}} \sin i=25-30 \mathrm{~km} \mathrm{~s}^{-1}$ determined here with the information on stellar luminosity and temperature, we find $P_{\text {rot }} \leq 3.0-3.5 \mathrm{~d}$. However, despite the presence of surface abundance inhomogeneities, HD 115226 does not exhibit a prominent variability in the ASAS (Pojmanski 2002) photometric time-series. At the 
same time, Hipparcos Epoch Photometry of this star (ESA 1997) shows a marginal variation with $P=3.61 \mathrm{~d}$ and an amplitude below 0.01 mag.

\section{Analysis of pulsational variability}

Large rotational broadening in the spectrum of HD 115226 significantly reduces the number of lines suitable for precise radial velocity measurements. Fortunately, the star shows strong lines of Pr III and Nd III, which exhibit pulsational variability in almost all known roAp stars (Kochukhov \& Ryabchikova 2001; Ryabchikova et al. 2007b). Using the VALD database (Kupka et al. 1999) and an improved Nd III line list published by Ryabchikova et al. (2006), we measured radial velocities of the absorption features of doubly-ionized REEs and the hydrogen line cores. We determined velocities with the centre-ofgravity method. Based on these measurements, the amplitudes and phases of variable lines were found by least-squares fitting of the cosine function $A \cos [2 \pi(t / P+\varphi)]$.

The radial velocity analysis of strongest rare-earth lines readily shows pulsational signal with the frequency $\approx 1.53 \mathrm{mHz}$. The cores of $\mathrm{H} \alpha$ and $\mathrm{H} \beta$, as well as weaker lines of doubly-ionized $\mathrm{Pr}, \mathrm{Nd}$, and Dy, also show variation with this frequency. Thus, our observations demonstrate that HD 115226 is a new roAp star, pulsating with a period typical of this class of oscillating stars. However, the radial velocity amplitude in HD 115226 significantly exceeds the average level of a few hundred $\mathrm{m} \mathrm{s}^{-1}$ seen in other roAp stars.

In order to determine pulsational characteristics of HD 115226 with a better precision, we analysed combined radial velocity measurements of H I (2 lines), Pr III (5 lines), and Nd III (17 lines). Corresponding amplitude spectra are presented in Fig. 2. Combined velocity of the two weak Dy III lines shows a higher noise level (lower panel in Fig. 2), therefore we disregard this REE ion in the period determination. The least-squares fit yields $P=10.880 \pm 0.031,10.852 \pm 0.016$ and $10.867 \pm 0.010 \mathrm{~min}$ for H I, Pr III, and Nd III, respectively. The weighted mean value of the pulsation period is thus $10.864 \pm 0.008 \mathrm{~min}$ (frequency $1.534 \mathrm{mHz}$ ). Figure 3 shows the average radial velocity curve of Nd III together with a sinusoid fit. Analysis of the residuals shows no evidence of additional frequencies with amplitudes above $110 \mathrm{~m} \mathrm{~s}^{-1}$. For HI and Pr III the corresponding noise level (amplitude of the highest noise peak in the residuals) is $160 \mathrm{~m} \mathrm{~s}^{-1}$. Further spectroscopic observations at a larger telescope are required to confirm monoperiodic character of oscillations in HD 115226.

Finally, we have derived a least-squares estimate of the amplitudes and phases of 26 variable lines for the fixed $10.864 \mathrm{~min}$ period (Table 2). Integration time of $120 \mathrm{~s}$ adopted in our observations of HD 115226 is non-negligible compared to the inferred pulsational period. We found that for the time sampling of our data the phase smearing reduces amplitudes by $6.2 \%$. Consequently, all radial velocity amplitudes reported here are multiplied by a factor 1.066 to correct for this effect.

From Table 2 it is evident that oscillation amplitudes show considerable scatter even for the lines of the same ion. This can be attributed to the dilution of pulsation signal in the lines affected by unrecognized blends. For this reason, our amplitude determination possibly gives only a lower limit of the real signal for many pulsating lines. Thus, we believe that the radial velocity amplitudes exceeding $1 \mathrm{~km} \mathrm{~s}^{-1}$, as seen for $\mathrm{Nd}$ III 4914, 5802, 5851,6327 , and $6550 \AA$ are more representative of the pulsations in HD 115226 than variability in the lines with amplitudes around $500 \mathrm{~m} \mathrm{~s}^{-1}$.
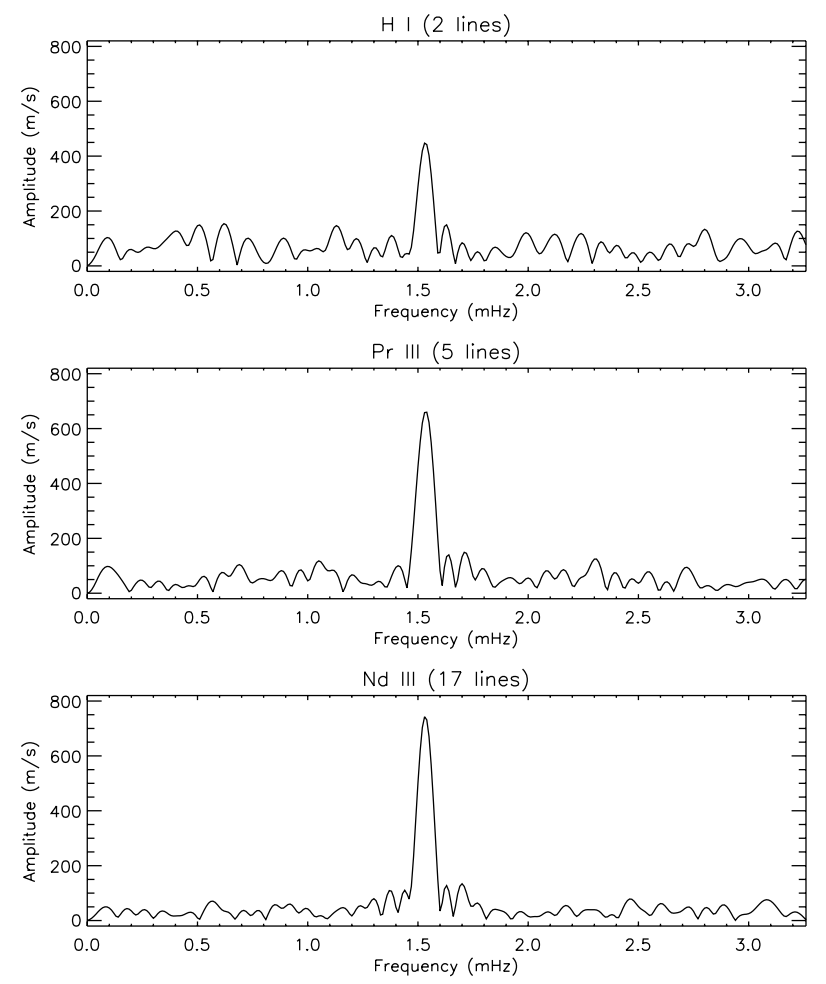

Dy III (2 lines)

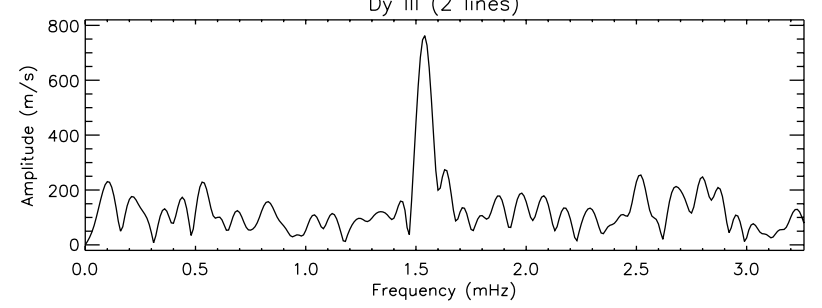

Fig. 2. Amplitude spectra for the combined radial velocity of two H I, 5 Pr III, $17 \mathrm{Nd} \mathrm{III,} \mathrm{and} \mathrm{two} \mathrm{Dy} \mathrm{III} \mathrm{lines.} \mathrm{The} \mathrm{presence} \mathrm{of} \mathrm{rapid} \mathrm{oscillation}$ with the frequency $1.53 \mathrm{mHz}$ is evident.
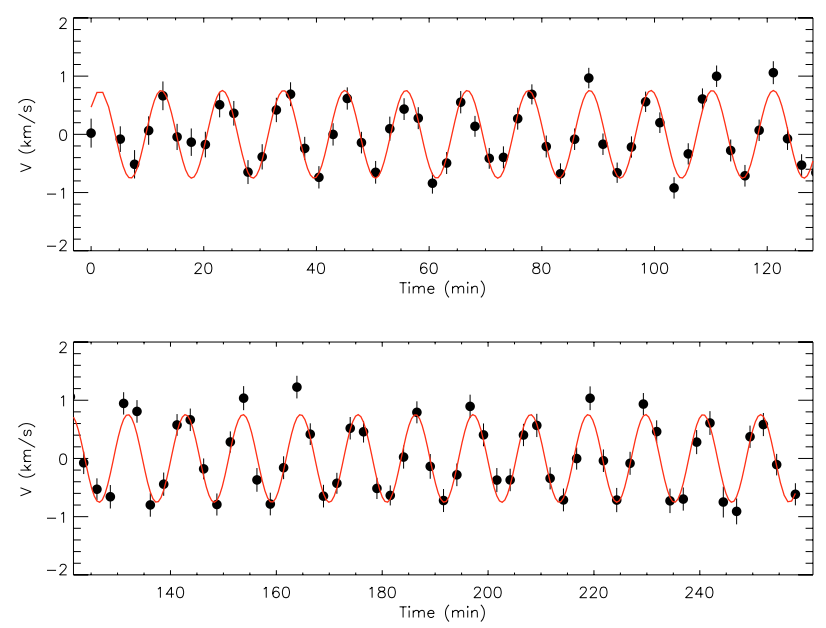

Fig. 3. Average radial velocity of $17 \mathrm{Nd}$ III lines as a function of time. Symbols show observations. The solid curve illustrates a least-squares cosine fit with $P=10.864 \mathrm{~min}$.

Time-series analysis reveals that the lines of Pr III and Nd III pulsate with approximately the same phase, which differs from that of Dy III and H I. In our model, a larger pulsation phase corresponds to an earlier radial velocity maximum. Therefore, we find that the Dy III velocity lags by 0.1 period behind HI. The phase lag reaches 0.2 for Pr III and Nd III. 
Table 2. Amplitudes and phases of individual lines of H I, Pr III, Nd III, and Dy III determined by the least-squares analysis of radial velocity measurements. The phases, determined with respect to the BJD of the first observation (see Sect. 2), are given in units of pulsation period.

\begin{tabular}{lrrc}
\hline \hline Ion & $\lambda(\AA)$ & $A\left(\mathrm{~m} \mathrm{~s}^{-1}\right)$ & $\varphi$ \\
\hline H I & 4861.32 & $423 \pm 81$ & $0.043 \pm 0.033$ \\
H I & 6562.78 & $526 \pm 58$ & $0.021 \pm 0.019$ \\
\hline Pr III & 5956.04 & $777 \pm 94$ & $0.820 \pm 0.021$ \\
Pr III & 6090.01 & $644 \pm 86$ & $0.837 \pm 0.023$ \\
Pr III & 6160.23 & $841 \pm 108$ & $0.795 \pm 0.022$ \\
Pr III & 6195.62 & $622 \pm 72$ & $0.764 \pm 0.020$ \\
Pr III & 6866.79 & $886 \pm 90$ & $0.871 \pm 0.017$ \\
\hline Nd III & 4759.53 & $616 \pm 117$ & $0.925 \pm 0.032$ \\
Nd III & 4769.62 & $714 \pm 101$ & $0.906 \pm 0.024$ \\
Nd III & 4911.65 & $620 \pm 63$ & $0.885 \pm 0.017$ \\
Nd III & 4914.09 & $1242 \pm 97$ & $0.870 \pm 0.013$ \\
Nd III & 4927.57 & $504 \pm 53$ & $0.833 \pm 0.018$ \\
Nd III & 4942.64 & $430 \pm 66$ & $0.831 \pm 0.026$ \\
Nd III & 5050.70 & $949 \pm 50$ & $0.853 \pm 0.009$ \\
Nd III & 5102.42 & $763 \pm 62$ & $0.791 \pm 0.014$ \\
Nd III & 5126.99 & $546 \pm 55$ & $0.809 \pm 0.017$ \\
Nd III & 5294.11 & $908 \pm 96$ & $0.778 \pm 0.018$ \\
Nd III & 5677.15 & $744 \pm 108$ & $0.863 \pm 0.025$ \\
Nd III & 5802.53 & $1183 \pm 173$ & $0.899 \pm 0.025$ \\
Nd III & 5845.07 & $554 \pm 71$ & $0.824 \pm 0.021$ \\
Nd III & 5851.54 & $1212 \pm 122$ & $0.872 \pm 0.017$ \\
Nd III & 6145.07 & $766 \pm 122$ & $0.855 \pm 0.027$ \\
Nd III & 6327.26 & $1011 \pm 108$ & $0.874 \pm 0.018$ \\
Nd III & 6550.24 & $1005 \pm 198$ & $0.786 \pm 0.033$ \\
\hline Dy III & 4510.03 & $789 \pm 82$ & $0.928 \pm 0.017$ \\
Dy III & 5730.28 & $823 \pm 130$ & $0.956 \pm 0.027$ \\
\hline & & &
\end{tabular}

We also searched a number of strong lines belonging to the iron-peak elements and $\mathrm{Ca}$ for pulsation signatures. We detected no variability exceeding $100 \mathrm{~m} \mathrm{~s}^{-1}$ in these lines.

\section{Discussion}

We show that HD 115226 is a new roAp star with broad lines and prominent pulsational variability in the cores of hydrogen lines and in doubly-ionized $\mathrm{Pr}, \mathrm{Nd}$, and Dy. The radial velocity amplitudes, exceeding $1 \mathrm{~km} \mathrm{~s}^{-1}$ in some $\mathrm{Nd}$ III lines are rather high for a roAp star. On the other hand, atmospheric parameters and chemical abundance pattern of HD 115226 are close to those of known roAp stars. The phase shifts between radial velocity variation of different spectral lines suggest the presence of running pulsation wave, first propagating in the atmospheric layers where hydrogen lines form and then reaching the heights where REEs are enhanced. This behaviour is observed in many other roAp stars (Ryabchikova et al. 2007b).

Recent studies reported pulsational radial velocity variations at the level below $100 \mathrm{~m} \mathrm{~s}^{-1}$ for HD 137909 (Hatzes \& Mkrtichian 2004), HD 116114 (Elkin et al. 2005b), HD 154708 (Kurtz et al. 2006), and HD 75445 (Kochukhov et al. 2007a). In contrast, our discovery of oscillations in HD 115226 adds to the list of roAp stars with much higher amplitudes. In fact, given the low probability of obtaining observations at the rotation phase of maximum pulsational variability, it is quite possible that HD 115226 has one of the highest radial velocity amplitudes among all roAp stars.

Rapid rotation and high pulsational amplitude makes HD 115226 similar to HD 83368 (Kochukhov 2006) and HD 99563 (Elkin et al. 2005a). Like these two well-studied roAp stars, HD 115226 is an obvious candidate for monitoring pulsations over a complete rotation cycle. We propose to obtain such observations both in photometry, to determine rotation period and to study the pulsation frequency spectrum, and in spectroscopy, to derive distribution of chemical spots and to study the topology of pulsation velocity field.

The discovery of strong variability in HD 115226 hints at the existence of a relationship between the stellar rotation and excitation of pulsations. The group of roAp stars that show radial velocity amplitudes in $\mathrm{Nd}$ III at the level of $1 \mathrm{~km} \mathrm{~s}^{-1}$ and above now includes HD 60435, HD 83368, HD 99563, HD 115226, HD 12932, and HD 19918. The first four stars all have $v_{\mathrm{e}} \sin i>10 \mathrm{~km} \mathrm{~s}^{-1}$ and $P_{\text {rot }}<8 \mathrm{~d}$. Rotation periods are unknown for the two latter stars, but cannot be long given $v_{\mathrm{e}} \sin i=3.0-3.5 \mathrm{~km} \mathrm{~s}^{-1}$ measured for these objects (Ryabchikova et al. 2007b). Thus, we come to the conclusion that the high-amplitude roAp stars are markedly different in their rotation properties from roAp stars with lower pulsational amplitudes, many of which have rotation periods of the order of years and even decades. In the light of this finding, the problem of the influence of rotation on excitation of $p$-modes in roAp stars deserves careful theoretical consideration.

Acknowledgements. This work was supported by the RFBI grant 06-02-16110a, RAS Presidium Program "Origin and Evolution of Stars and Galaxies", and the Austrian Science Fund (FWF projects P17580, P17890).

\section{References}

Elkin, V. G., Kurtz, D. W., \& Mathys, G. 2005a, MNRAS, 364, 864

Elkin, V. G., Riley, J. D., Cunha, M. S., Kurtz, D. W., \& Mathys, G. 2005b, MNRAS, 358, 665

ESA 1997, ESA Special Publication, 1200, The HIPPARCOS and TYCHO catalogues

Gomez, A. E., Luri, X., Grenier, S., et al. 1998, A\&A, 336, 953

Hatzes, A. P., \& Mkrtichian, D. E. 2004, MNRAS, 351, 663

Kochukhov, O. 2004, ApJ, 615, L149

Kochukhov, O. 2006, A\&A, 446, 1051

Kochukhov, O. 2007a, Commun. Asteroseismol., 150, 39

Kochukhov, O. 2007b, in Physics of Magnetic Stars, ed. I. I. Romanyuk, D. O. Kudryavtsev, 109

Kochukhov, O., \& Bagnulo, S. 2006, A\&A, 450, 763

Kochukhov, O., \& Ryabchikova, T. 2001, A\&A, 374, 615

Kochukhov, O., Drake, N. A., Piskunov, N., \& de la Reza, R. 2004, A\&A, 424, 935

Kochukhov, O., Ryabchikova, T., Bagnulo, S., \& Lo Curto, G. 2007a, in CP\#Ap Workshop, ed. J.Žižňovský, J. Zverko, E. Paunzen, \& M. Netopil, Contribut. Astr. Obs. Skalnaté Pleso, in press [arXiv:0711.4923]

Kochukhov, O., Ryabchikova, T., Weiss, W. W., Landstreet, J. D., \& Lyashko, D. $2007 b$, MNRAS, 376, 651

Kupka, F., Piskunov, N., Ryabchikova, T. A., Stempels, H. C., \& Weiss, W. W. 1999, A\&AS, 138, 119

Kurtz, D. W., \& Martinez, P. 2000, Baltic Astron., 9, 253

Kurtz, D. W., Elkin, V. G., Cunha, M. S., et al. 2006, MNRAS, 372, 286

Martinez, P. 1993, Ph.D. Thesis, University of Cape Town, SA

Mayor, M., Pepe, F., Queloz, D., et al. 2003, The Messenger, 114, 20

Mkrtichian, D. E., Hatzes, A. P., \& Kanaan, A. 2003, MNRAS, 345, 781

Moon, T. T., \& Dworetsky, M. M. 1985, MNRAS, 217, 305

Napiwotzki, R., Schoenberner, D., \& Wenske, V. 1993, A\&A, 268, 653

Pojmanski, G. 2002, AcA, 52, 397

Renson, P., Gerbaldi, M., \& Catalano, F. A. 1991, A\&AS, 89, 429

Ryabchikova, T. A., Savanov, I. S., Malanushenko, V. P., \& Kudryavtsev, D. O. 2001, Astron. Rep., 45, 382

Ryabchikova, T., Piskunov, N., Kochukhov, O., et al. 2002, A\&A, 384, 545

Ryabchikova, T., Nesvacil, N., Weiss, W. W., Kochukhov, O., \& Stütz, C. 2004, A\&A, 423, 705

Ryabchikova, T., Ryabtsev, A., Kochukhov, O., \& Bagnulo, S. 2006, A\&A, 456, 329

Ryabchikova, T., Kochukhov, O., \& Bagnulo, S. 2007a, in Physics of Magnetic Stars, ed. I. I. Romanyuk, \& D. O. Kudryavtsev, 325

Ryabchikova, T., Sachkov, M., Kochukhov, O., \& Lyashko, D. 2007b, A\&A, 473, 907

Savanov, I. S., Malanushenko, V. P., \& Ryabchikova, T. A. 1999, Astron. Lett., 25,802

Schaller, G., Schaerer, D., Meynet, G., \& Maeder, A. 1992, A\&AS, 96, 269

Vauclair, S., \& Théado, S. 2004, A\&A, 425, 179 